\title{
Analysis of a frictionless contact problem for elastic bodies
}

\author{
by S. Drabla (Sétif), M. Sofonea (Perpignan) and \\ B. Teniou (Constantine)
}

\begin{abstract}
This paper deals with a nonlinear problem modelling the contact between an elastic body and a rigid foundation. The elastic constitutive law is assumed to be nonlinear and the contact is modelled by the well-known Signorini conditions. Two weak formulations of the model are presented and existence and uniqueness results are established using classical arguments of elliptic variational inequalities. Some equivalence results are presented and a strong convergence result involving a penalized problem is also proved.
\end{abstract}

1. Introduction. In this paper we consider the following physical setting. An elastic body is being acted upon by given forces and surface tractions and it is in contact with a rigid foundation. The contact is frictionless and we assume that a loss of contact may occur. By assumption the forces change slowly in time so that the accelerations in the system are negligible. Thus, we neglect the inertial terms in the equations of motion and we obtain a static approximation for the process.

The elastic body occupies the domain $\Omega \subset \mathbb{R}^{N}(N=1,2,3)$ and we denote its surface by $\Gamma$. We assume that $\Gamma$ is partitioned into three disjoint measurable parts $\Gamma_{1}, \Gamma_{2}$ and $\Gamma_{3}$ such that meas $\Gamma_{1}>0$. We assume that the body is clamped on $\Gamma_{1}$ and thus the displacement field vanishes there and that surface tractions $\varphi_{2}$ act on $\Gamma_{2}$. The solid is in unilateral frictionless contact with a rigid foundation on $\Gamma_{3}$, which means that the body and the foundation have a compliant shape on $\Gamma_{3}$. Assuming that a volume force of density $\varphi_{1}$ is applied on $\Omega$, the mechanical problem may be formulated classically as follows:

Problem $P$. Find a displacement field $u=\left(u_{i}\right): \Omega \rightarrow \mathbb{R}^{N}$ and a stress field $\sigma=\left(\sigma_{i j}\right): \Omega \rightarrow S_{N}$ such that

1991 Mathematics Subject Classification: 73C35, 73V25, 49J40, $49 \mathrm{~J} 45$.

Key words and phrases: frictionless contact, elastic body, variational inequality, monotone operator, penalized problem. 


$$
\begin{aligned}
& \sigma=F(\varepsilon(u)) \quad \text { in } \Omega, \\
& \operatorname{Div} \sigma+\varphi_{1}=0 \quad \text { in } \Omega \text {, } \\
& u=0 \quad \text { on } \Gamma_{1} \text {, } \\
& \sigma \nu=\varphi_{2} \quad \text { on } \Gamma_{2} \text {, } \\
& u_{\nu} \leq 0, \quad \sigma_{\nu} \leq 0, \quad \sigma_{\tau i}=0, \quad \sigma_{\nu} u_{\nu}=0 \quad \text { on } \Gamma_{3} \text {. }
\end{aligned}
$$

Here and below $i, j=1, \ldots, N$ and summation over repeated indices is implied. Moreover, $S_{N}$ denotes the set of second order symmetric tensors on $\mathbb{R}^{N}$. The equation (1.1) is the elastic constitutitve law in which $\varepsilon(u)$ is the small strain tensor and $F$ is a given nonlinear function while (1.2) is the equilibrium equation, since the inertial term has been omitted. The boundary conditions (1.3) and (1.4) are the displacement-tractions boundary conditions in which $\nu=\left(\nu_{i}\right)$ represents the outward unit normal vector on $\Gamma$ while (1.5) are the classical Signorini contact conditions where

$$
u_{\nu}=u_{i} \nu_{i}, \quad \sigma_{\nu}=\sigma_{i j} \nu_{j} \nu_{i}, \quad \sigma_{\tau i}=\sigma_{i j} \nu_{j}-\sigma_{\nu} \nu_{i} .
$$

Similar frictionless contact problems were already studied for elastic or viscoelastic bodies (see for instance [2], [5]-[9], [12] and the references therein), as well as for elasto-visco-plastic bodies (see for instance [3], [14] and [15]).

The aim of this paper is to extend some known existence and uniqueness results to the case of the nonlinear constitutive law (1.1) and to point out some new results concerning the mechanical problem (1.1)-(1.5). The paper is organized as follows. In Section 2 some notation and functional preliminaries are presented while in Section 3 two variational formulations $P_{1}$ and $P_{2}$ are established. Problem $P_{1}$ is obtained from (1.1)-(1.5) using a Green type formula and the constitutive law. Since in this way the stress field is eliminated, the unknown of this problem is the displacement field $u$. Problem $P_{2}$ is also obtained from (1.1)-(1.5) using a similar method. Since in this case the displacement field is eliminated, problem $P_{2}$ involves as unknown only the stress field $\sigma$.

In Section 4 we give existence and uniqueness results for problems $P_{1}$ and $P_{2}$, using classical arguments of elliptic variational inequalities. We also study the link between the solutions of the variational problems $P_{1}$ and $P_{2}$ and we show that if $u$ denotes the solution of $P_{1}$ and $\sigma$ is the solution of $P_{2}$ then $\sigma$ and $u$ are related by the elastic constitutive law (1.1). The last section deals with a penalized problem governed by a parameter $h>0$. For this problem we prove existence and uniqueness results and we give a convergence result as $h \rightarrow 0$, in order to make the link with the mechanical problem (1.1)-(1.5). 
2. Notation and preliminaries. We start this section by presenting the notation we shall use and some preliminary material. For further details we refer the reader to [4], [10] or [13]. We denote by "." the inner product in the spaces $\mathbb{R}^{N}$ and $S_{N}$ and by $|\cdot|$ the Euclidean norm on these spaces. We also use the following notation:

$$
\begin{aligned}
H & =\left\{u=\left(u_{i}\right) \mid u_{i} \in L^{2}(\Omega)\right\}, \\
\mathcal{H} & =\left\{\sigma=\left(\sigma_{i j}\right) \mid \sigma_{i j}=\sigma_{j i} \in L^{2}(\Omega)\right\}, \\
H_{1} & =\left\{u=\left(u_{i}\right) \mid u_{i} \in H^{1}(\Omega)\right\}, \\
\mathcal{H}_{1} & =\left\{\sigma \in \mathcal{H} \mid \sigma_{i j, j} \in H\right\} .
\end{aligned}
$$

$H, \mathcal{H}, H_{1}$ and $\mathcal{H}_{1}$ are real Hilbert spaces endowed with the inner products given by

$$
\begin{gathered}
\langle u, v\rangle_{H}=\int_{\Omega} u_{i} v_{i} d x, \quad\langle\sigma, \tau\rangle_{\mathcal{H}}=\int_{\Omega} \sigma_{i j} \tau_{i j} d x, \\
\langle u, v\rangle_{H_{1}}=\langle u, v\rangle_{H}+\langle\varepsilon(u), \varepsilon(v)\rangle_{\mathcal{H}}, \\
\langle\sigma, \tau\rangle_{\mathcal{H}_{1}}=\langle\sigma, \tau\rangle_{\mathcal{H}}+\langle\operatorname{Div} \sigma, \operatorname{Div} \tau\rangle_{H},
\end{gathered}
$$

where $\varepsilon: H_{1} \rightarrow \mathcal{H}$ and Div $: \mathcal{H}_{1} \rightarrow H$ are the deformation and divergence operators, respectively, defined by

$$
\varepsilon(v)=\left(\varepsilon_{i j}(v)\right), \quad \varepsilon_{i j}(v)=\frac{1}{2}\left(v_{i, j}+v_{j, i}\right), \quad \operatorname{Div} \sigma=\left(\sigma_{i j, j}\right) .
$$

The associated norms on $H, \mathcal{H}, H_{1}$ and $\mathcal{H}_{1}$ are denoted by $|\cdot|_{H},|\cdot|_{\mathcal{H}},|\cdot|_{H_{1}}$ and $|\cdot|_{\mathcal{H}_{1}}$, respectively.

Let $H_{\Gamma}=H^{1 / 2}(\Gamma)^{N}$ and let $\gamma: H_{1} \rightarrow H_{\Gamma}$ be the trace map. For every $v \in H_{1}$ we use, when no confusion is likely, the notation $v$ for the trace $\gamma v$ of $v$ on $\Gamma$ and we denote by $v_{\nu}$ and $v_{\tau}$ the normal and tangential components of $v$ on $\Gamma$ given by

$$
v_{\nu}=v \cdot \nu, \quad v_{\tau}=v-v_{\nu} \nu .
$$

Let $V$ denote the closed subspace of $H_{1}$ defined by

$$
V=\left\{v \in H_{1} \mid v=0 \text { on } \Gamma_{1}\right\} .
$$

Since meas $\Gamma_{1}>0$, Korn's inequality holds:

$$
|\varepsilon(v)|_{\mathcal{H}} \geq C|v|_{H_{1}} \quad \forall v \in V ;
$$

see, e.g., [8], p. 79. Here and below, $C$ denotes a strictly positive generic constant which may depend on $\Omega, \Gamma_{1}, \Gamma_{2}, \Gamma_{3}$, and $F$, but does not depend on the input data $\varphi_{1}, \varphi_{2}$ and whose value may vary from place to place.

On $V$ we consider the inner product given by

$$
\langle u, v\rangle_{V}=\langle\varepsilon(u), \varepsilon(v)\rangle_{\mathcal{H}}
$$


Using (2.3) we see that $|\cdot|_{H_{1}}$ and $|\cdot|_{V}$ are equivalent norms on $V$. Therefore $\left(V,|\cdot|_{V}\right)$ is a real Hilbert space.

Let $H_{\Gamma}^{\prime}$ be the dual of $H_{\Gamma}$ and let $\langle\cdot, \cdot\rangle$ denote the duality pairing between $H_{\Gamma}^{\prime}$ and $H_{\Gamma}$. For every $\sigma \in \mathcal{H}_{1}$ let $\sigma \nu$ be the element of $H_{\Gamma}^{\prime}$ given by

$$
\langle\sigma \nu, \gamma v\rangle=\langle\sigma, \varepsilon(v)\rangle_{\mathcal{H}}+\langle\operatorname{Div} \sigma, v\rangle_{H} \quad \forall v \in H_{1} .
$$

We recall that if $\sigma$ is a regular function (say $C^{1}$ ), then

$$
\langle\sigma \nu, \gamma v\rangle=\int_{\Gamma} \sigma \nu \cdot v d a \quad \forall v \in H_{1}
$$

where $d a$ is the surface measure element.

In the study of the mechanical problem (1.1)-(1.5) we consider the following assumptions:

$$
\begin{aligned}
& \left\{\begin{array}{l}
F: \Omega \times S_{N} \rightarrow S_{N} \text { and } \\
\text { (a) there exists } M>0 \text { such that }
\end{array}\right. \\
& \left|F\left(x, \varepsilon_{1}\right)-F\left(x, \varepsilon_{2}\right)\right| \leq M\left|\varepsilon_{1}-\varepsilon_{2}\right| \\
& \text { for all } \varepsilon_{1}, \varepsilon_{2} \in S_{N} \text {, a.e. in } \Omega \text {; } \\
& \text { (b) there exists } m>0 \text { such that } \\
& \left.\left(F\left(x, \varepsilon_{1}\right)\right)-F\left(x, \varepsilon_{2}\right)\right) \cdot\left(\varepsilon_{1}-\varepsilon_{2}\right) \geq m\left|\varepsilon_{1}-\varepsilon_{2}\right|^{2} \\
& \text { for all } \varepsilon_{1}, \varepsilon_{2} \in S_{N} \text {, a.e. in } \Omega \text {; } \\
& \text { (c) } x \mapsto F(x, \varepsilon) \text { is Lebesgue measurable on } \Omega \text { for all } \varepsilon \in S_{N} \text {; } \\
& \text { (d) } x \mapsto F(x, 0) \in \mathcal{H} \text {, } \\
& \varphi_{1} \in H, \\
& \varphi_{2} \in L^{2}\left(\Gamma_{2}\right)^{N} .
\end{aligned}
$$

REMARK 2.1. Using (2.7) we find that for all $\tau \in \mathcal{H}$ the function $x \mapsto$ $F(x, \tau(x))$ belongs to $\mathcal{H}$ and hence we may consider $F$ as an operator defined on $\mathcal{H}$ with range in $\mathcal{H}$. Moreover, $F: \mathcal{H} \rightarrow \mathcal{H}$ is a strongly monotone Lipschitz continuous operator and therefore $F$ is invertible and its inverse $F^{-1}: \mathcal{H} \rightarrow \mathcal{H}$ is also a strongly monotone Lipschitz continuous operator.

Using (2.8), (2.9) and the Riesz representation theorem we can consider the element $f \in V$ given by

$$
\langle f, v\rangle_{V}=\left\langle\varphi_{1}, v\right\rangle_{H}+\left\langle\varphi_{2}, \gamma v\right\rangle_{L^{2}\left(\Gamma_{2}\right)^{N}} \quad \forall v \in V .
$$

Finally, we denote in the sequel by $U$ the set of geometrically admissible displacement fields defined by

$$
U=\left\{v \in V \mid v_{\nu} \leq 0 \text { on } \Gamma_{3}\right\}
$$

and by $\Sigma$ the set of statically admissible stress fields given by

$$
\Sigma=\left\{\tau \in \mathcal{H} \mid\langle\tau, \varepsilon(v)\rangle_{\mathcal{H}} \geq\langle f, v\rangle_{V} \forall v \in U\right\} .
$$


3. Variational formulations. In this section we deduce two variational formulations for the mechanical problem (1.1)-(1.5). We suppose in the sequel that (2.7)-(2.9) hold. We have the following result:

LEMMA 3.1. If $\{u, \sigma\}$ are sufficiently regular functions satisfying (1.1)-(1.5), then

$$
\begin{gathered}
u \in U, \quad \sigma \in \Sigma \\
\langle\sigma, \varepsilon(v)-\varepsilon(u)\rangle_{\mathcal{H}} \geq\langle f, v-u\rangle_{V} \quad \forall v \in U, \\
\langle\tau-\sigma, \varepsilon(u)\rangle_{\mathcal{H}} \geq 0 \quad \forall \tau \in \Sigma .
\end{gathered}
$$

Proof. The regularity $u \in U$ follows from (1.3), (1.5), (2.2) and (2.11). Let $v \in U$. Using (2.5), (2.6), (1.2)-(1.4) we have

$$
\langle\sigma, \varepsilon(v)\rangle_{\mathcal{H}}=\left\langle\varphi_{1}, v\right\rangle_{H}+\left\langle\varphi_{2}, \gamma v\right\rangle_{L^{2}\left(\Gamma_{2}\right)^{N}}+\int_{\Gamma_{3}} \sigma \nu \cdot v d a
$$

and using (1.5), (2.11) gives

$$
\int_{\Gamma_{3}} \sigma \nu \cdot v d a \geq 0
$$

So, by (3.4), (3.5) and (2.10) we deduce $\langle\sigma, \varepsilon(v)\rangle_{\mathcal{H}} \geq\langle f, v\rangle_{V}$, i.e. $\sigma \in \Sigma$.

Let us now remark that from (1.5) we have

$$
\int_{\Gamma_{3}} \sigma \nu \cdot u d a=0
$$

and taking $v=u$ in (3.4) and using again (2.10) we deduce

$$
\langle\sigma, \varepsilon(u)\rangle_{\mathcal{H}}=\langle f, u\rangle_{V} .
$$

The inequalities (3.2) and (3.3) are now a consequence of (2.12) and (3.6).

Lemma 3.1, (1.1) and Remark 2.1 allow us to consider the following two variational problems:

Problem $P_{1}$. Find a displacement field $u: \Omega \rightarrow \mathbb{R}^{N}$ such that

$$
u \in U, \quad\langle F(\varepsilon(u)), \varepsilon(v)-\varepsilon(u)\rangle_{\mathcal{H}} \geq\langle f, v-u\rangle_{V} \quad \forall v \in U .
$$

Problem $P_{2}$. Find a stress field $\sigma: \Omega \rightarrow S_{N}$ such that

$$
\sigma \in \Sigma, \quad\left\langle F^{-1}(\sigma), \tau-\sigma\right\rangle_{\mathcal{H}} \geq 0 \quad \forall \tau \in \Sigma .
$$

Remark 3.2. Problems $P_{1}$ and $P_{2}$ are formally equivalent to problem $P$. Indeed, if $u$ represents a regular solution of the variational problem $P_{1}$ and $\sigma$ is defined by $\sigma=F(\varepsilon(u)$ ), then using the arguments of [4] it follows that $\{u, \sigma\}$ is a solution of the mechanical problem $P$. Similarly, if $\sigma$ represents a regular solution of $P_{2}$ and $u \in V$ is given by $\sigma=F(\varepsilon(u))$ then, using the same arguments, it follows that $\{u, \sigma\}$ is a solution of $P$. For this reason 
we may consider problems $P_{1}$ and $P_{2}$ as variational formulations of the mechanical problem $P$.

4. Existence and uniqueness results. In this section we present existence and uniqueness results for the variational problems $P_{1}$ and $P_{2}$ and we study the link between the solutions of these problems.

THEOREM 4.1. Let (2.7)-(2.9) hold. Then there exists a unique solution $u$ of problem $P_{1}$ having the regularity $u \in V$.

Proof. Using Riesz's representation theorem we may define the operator $A: V \rightarrow V$ by

$$
\langle A w, v\rangle_{V}=\langle F(\varepsilon(w)), \varepsilon(v)\rangle_{\mathcal{H}} \quad \forall w, v \in V .
$$

From (2.7)(a) it results that $A$ is a Lipschitz continuous operator and using (2.7)(b) and Korn's inequality (2.3) we find that $A$ is a strongly monotone operator. Moreover, by (2.11), $U$ is a closed convex nonempty subset of $V$. Theorem 4.1 now results from (4.1) and standard arguments of elliptic variational inequalities (see e.g. [1] or [11]).

THEOREM 4.2. Let (2.7)-(2.9) hold. Then there exists a unique solution $\sigma$ of problem $P_{2}$ having the regularity $\sigma \in \mathcal{H}_{1}$.

Proof. By (2.12), $\Sigma$ is a closed convex subset of $\mathcal{H}$ and from (2.4) it follows that $\varepsilon(f) \in \Sigma$, i.e. $\Sigma$ is nonempty. Moreover, by Remark 2.1, $F^{-1}: \mathcal{H} \rightarrow \mathcal{H}$ is a strongly monotone Lipschitz continuous operator. Using again classical arguments of elliptic variational inequalities we obtain the existence and uniqueness of a solution $\sigma \in \Sigma$ of (3.8). Moreover, upon taking $v= \pm \varphi$ with $\varphi \in \mathcal{D}(\Omega)^{N}$, from (2.12) it follows that

$$
\langle\sigma, \varepsilon(\varphi)\rangle_{\mathcal{D}^{\prime}(\Omega)^{N} \times \mathcal{D}(\Omega)^{N}}=\langle f, \varphi\rangle_{H}
$$

and using (2.10) we obtain

$$
\operatorname{Div} \sigma+\varphi_{1}=0 \quad \text { a.e. in } \Omega \text {. }
$$

Using now (2.8), we deduce $\sigma \in \mathcal{H}_{1}$, which proves Theorem 4.2.

In the sequel we study the link between the solutions $u$ and $\sigma$ of the variational problems $P_{1}$ and $P_{2}$. We have the following result:

Theorem 4.3. Let (2.7)-(2.9) hold and let $u \in V, \sigma \in \mathcal{H}_{1}$. Consider the following properties:

(i) $u$ is the solution of problem $P_{1}$ given in Theorem 4.1;

(ii) $\sigma$ is the solution of problem $P_{2}$ given in Theorem 4.2;

(iii) $\sigma$ and $u$ are connected by the elastic constitutive law (1.1).

Then, if two of the above properties hold, so does the remaining one. 
Pr o of. (i) + (iii) $\Rightarrow$ (ii). Suppose that (i) and (iii) hold. Using Remark 2.1, (1.1) and (3.7) we have $\sigma \in \mathcal{H}$ and

$$
\langle\sigma, \varepsilon(v)-\varepsilon(u)\rangle_{\mathcal{H}} \geq\langle f, v-u\rangle_{V} \quad \forall v \in U
$$

Taking now $v=2 u$ and $v=0$ in (4.2) gives

$$
\langle\sigma, \varepsilon(u)\rangle_{\mathcal{H}}=\langle f, u\rangle_{V}
$$

So, from (4.2), (4.3) we obtain $\langle\sigma, \varepsilon(v)\rangle_{\mathcal{H}} \geq\langle f, v\rangle_{V}$ for all $v \in U$, which shows that

$$
\sigma \in \Sigma
$$

Using the same arguments as in the proof of Theorem 4.2, from (4.4) we deduce $\sigma \in \mathcal{H}_{1}$. Moreover, for all $\tau \in \Sigma$, using (1.1), (2.12) and (4.3) we have

$$
\left\langle F^{-1}(\sigma), \tau-\sigma\right\rangle_{\mathcal{H}}=\langle\tau-\sigma, \varepsilon(u)\rangle_{\mathcal{H}} \geq 0 .
$$

Finally, from (4.4) and (4.5) we conclude that $\sigma$ is a solution of problem $P_{2}$.

(i) + (ii) $\Rightarrow$ (iii). Suppose that (i) and (ii) hold and define

$$
\tilde{\sigma}=F(\varepsilon(u)) \in \mathcal{H} .
$$

By the previous step, $\widetilde{\sigma}$ is a solution of problem $P_{2}$. The uniqueness part of Theorem 4.2 shows that

$$
\sigma=\widetilde{\sigma}
$$

So, from (4.7) and (4.6) we obtain (iii).

(ii) + (iii) $\Rightarrow$ (i). Suppose that (ii) and (iii) hold and set

$$
\begin{gathered}
\Sigma_{0}=\left\{\tau \in \mathcal{H} \mid\langle\tau, \varepsilon(v)\rangle_{\mathcal{H}} \geq 0 \forall v \in U\right\}, \\
\widetilde{\sigma}=\varepsilon(f), \\
\sigma_{0}=\sigma-\widetilde{\sigma} .
\end{gathered}
$$

Using (4.8)-(4.10), (2.12) and (2.4) it is easy to see that

$$
\sigma \in \Sigma \Leftrightarrow \sigma_{0} \in \Sigma_{0} .
$$

So, using (3.8), (4.11) and (1.1) we have

$$
\left\langle\tau-\sigma_{0}, \varepsilon(u)\right\rangle_{\mathcal{H}} \geq 0 \quad \forall \tau \in \Sigma_{0} .
$$

We now prove that $u \in U$. Suppose that $u \notin U$ and let $P u$ denote the projection of $u$ on the closed convex set $U \subset V$. We have

$$
\left.\langle P u-u, v\rangle_{V} \geq\langle P u-u, P u\rangle_{V}\right\rangle\langle P u-u, u\rangle_{V} \quad \forall v \in U .
$$

So, there exists $\alpha \in \mathbb{R}$ such that

$$
\langle P u-u, v\rangle_{V}>\alpha>\langle P u-u, u\rangle_{V} \quad \forall v \in U .
$$


Let

$$
\widetilde{\tau}=\varepsilon(P u-u) \in \mathcal{H} .
$$

Using (2.4), (4.13) and (4.14) we have

$$
\langle\widetilde{\tau}, \varepsilon(v)\rangle_{\mathcal{H}}>\alpha>\langle\widetilde{\tau}, \varepsilon(u)\rangle_{\mathcal{H}} \quad \forall v \in U .
$$

Taking $v=0$ in (4.15) we obtain

$$
\alpha<0 \text {. }
$$

Now suppose that there exists $\widetilde{v} \in U$ such that

$$
\langle\widetilde{\tau}, \varepsilon(\widetilde{v})\rangle_{\mathcal{H}}<0 .
$$

Using (4.15) for $\lambda \widetilde{v} \in U$ with $\lambda \geq 0$ we have

$$
\lambda\langle\widetilde{\tau}, \varepsilon(\widetilde{v})\rangle_{\mathcal{H}}>\alpha \quad \forall \lambda \geq 0 .
$$

So, passing to the limit as $\lambda \rightarrow+\infty$, in (4.18) and using (4.17) we deduce $\alpha \leq-\infty$, which contradicts $\alpha \in \mathbb{R}$. So, we proved that

$$
\langle\widetilde{\tau}, \varepsilon(v)\rangle_{\mathcal{H}} \geq 0 \quad \forall v \in U
$$

and using (4.8) we have $\widetilde{\tau} \in \Sigma_{0}$. Using now (4.12) we deduce

$$
\langle\widetilde{\tau}, \varepsilon(u)\rangle_{\mathcal{H}} \geq\left\langle\sigma_{0}, \varepsilon(u)\right\rangle_{\mathcal{H}} .
$$

So, by (4.19), (4.15) and (4.16) we obtain

$$
\left\langle\sigma_{0}, \varepsilon(u)\right\rangle_{\mathcal{H}}<0 .
$$

Moreover, taking $\tau=2 \sigma_{0} \in \Sigma_{0}$ in (4.12) gives

$$
\left\langle\sigma_{0}, \varepsilon(u)\right\rangle_{\mathcal{H}} \geq 0 .
$$

As (4.20) and (4.21) contradict each other, it results that $u \in U$.

Using now (4.9) and (2.4) we have $\widetilde{\sigma} \in \Sigma$. So, taking $\tau=\widetilde{\sigma}$ in (3.8) and using again (2.4) we obtain

$$
\langle f, u\rangle_{V} \geq\langle\sigma, \varepsilon(u)\rangle_{\mathcal{H}} .
$$

Moreover, since $\sigma \in \Sigma$ and $u \in U$, by (2.12) we have

$$
\langle\sigma, \varepsilon(u)\rangle_{\mathcal{H}} \geq\langle f, u\rangle_{V} .
$$

So, by (4.22) and (4.23) we deduce

$$
\langle\sigma, \varepsilon(u)\rangle_{\mathcal{H}}=\langle f, u\rangle_{V}
$$

From (2.12) and (4.24) it now follows that

$$
\langle\sigma, \varepsilon(v)-\varepsilon(u)\rangle_{\mathcal{H}} \geq\langle f, v-u\rangle_{V} \quad \forall v \in V .
$$

Finally, using (1.1) and (4.25) we conclude that $u$ is a solution of problem $P_{1}$.

REMARK 4.4. The mechanical interpretation of the result obtained in Theorem 4.3 is the following: 
1) if the displacement field $u$ is the solution of the variational problem $P_{1}$ then the stress field $\sigma$ associated with $u$ by the elastic constitutive law $\sigma=F(\varepsilon(u))$ is the solution of the variational problem $P_{2}$;

2 ) if the stress field $\sigma$ is the solution of the variational problem $P_{2}$ then the displacement field $u \in V$ associated with $\sigma$ by the elastic constitutive law $\sigma=F(\varepsilon(u))$ is the solution of the variational problem $P_{1}$;

$3)$ if the displacement field $u$ is the solution of the problem $P_{1}$ and the stress field $\sigma$ is the solution of the problem $P_{2}$ then $u$ and $\sigma$ are connected by the elastic constitutive law $\sigma=F(\varepsilon(u))$.

For this reason we shall consider in the sequel the couple $\{u, \sigma\}$ given by Theorems 4.1 and 4.2 as a weak solution for the mechanical problem (1.1)-(1.5).

5. A strong convergence result. In this section we introduce a penalized problem for the contact problem (1.1)-(1.5) for which we give again two variational formulations and two existence and uniqueness results. Moreover, denoting by $\left\{u_{h}, \sigma_{h}\right\}$ the weak solution of this penalized problem depending on the parameter $h>0$, we obtain the strong convergence of $\left\{u_{h}, \sigma_{h}\right\}$ to the weak solution $\{u, \sigma\}$ of the original problem $P$ as $h \rightarrow 0$.

More precisely, let $h>0$. We consider the following boundary value problem:

Problem $P^{h}$. Find a displacement field $u_{h}: \Omega \rightarrow \mathbb{R}^{N}$ and a stress field $\sigma_{h}: \Omega \rightarrow S_{N}$ such that

$$
\begin{aligned}
& \sigma_{h}=F\left(\varepsilon\left(u_{h}\right)\right) \quad \text { in } \Omega \text {, } \\
& \operatorname{Div} \sigma_{h}+\varphi_{1}=0 \quad \text { in } \Omega \text {, } \\
& u_{h}=0 \quad \text { on } \Gamma_{1} \text {, } \\
& \sigma_{h} \nu=\varphi_{2} \quad \text { on } \Gamma_{2}, \\
& \left\{\begin{array}{l}
u_{h \nu} \leq 0 \Rightarrow \sigma_{h \nu}=0 \\
u_{h \nu}=0 \Rightarrow-1 / h<\sigma_{h \nu}<0, \quad \sigma_{h \tau i}=0 \quad \text { on } \Gamma_{3} . \\
u_{h \nu}>0 \Rightarrow \sigma_{h \nu}=-1 / h,
\end{array}\right.
\end{aligned}
$$

Let us remark that problem $P^{h}$ is similar to problem $P$ except that the Signorini frictionless contact conditions (1.5) are replaced by the contact conditions (5.5) which represent a penalization of (1.5). Indeed, formally, (5.5) becomes (1.5) as $h \rightarrow 0$, which is the main ingredient of the penalization. From the mechanical point of view, (5.5) represents a unilateral frictionless contact condition with a deformable foundation. The penetration of the elastic body and the foundation is possible and it occurs when the normal stress reaches the yield $1 / h$ where $h$ may be interpreted as the deformability coefficient of the foundation. 
In the study of the penalized problem (5.1)-(5.5) we suppose that (2.7)-(2.9) hold and consider the functions $\psi: \mathbb{R} \rightarrow \mathbb{R}$ and $j: V \rightarrow \mathbb{R}$ given by

$$
\begin{gathered}
\psi(x)= \begin{cases}0 & \text { if } x \leq 0, \\
x & \text { if } x>0,\end{cases} \\
j(v)=\int_{\Gamma_{3}} \psi\left(v_{\nu}\right) d a \quad \forall v \in V .
\end{gathered}
$$

Let $h>0$ and let

$$
\Sigma_{h}=\left\{\tau \in \mathcal{H} \mid\langle\tau, \varepsilon(v)\rangle_{\mathcal{H}}+\frac{1}{h} j(v) \geq\langle f, v\rangle_{V} \forall v \in V\right\}
$$

where $f$ is defined by (2.10).

Using the same arguments as in the proof of Lemma 3.1 we can give the following two variational formulations for the mechanical problem $P^{h}$ :

Problem $P_{1}^{h}$. Find a displacement field $u_{h}: \Omega \rightarrow \mathbb{R}^{N}$ such that

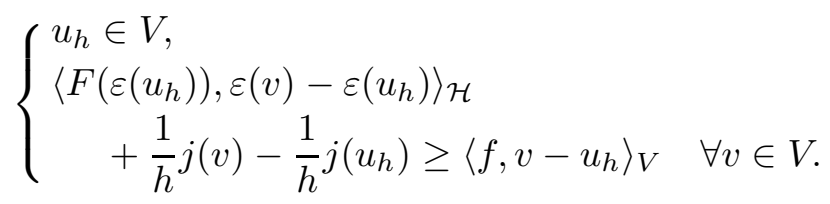

Problem $P_{2}^{h}$. Find a stress field $\sigma_{h}: \Omega \rightarrow S_{N}$ such that

$$
\sigma_{h} \in \Sigma_{h}, \quad\left\langle F^{-1}\left(\sigma_{h}\right), \tau-\sigma_{h}\right\rangle_{\mathcal{H}} \geq 0 \quad \forall \tau \in \Sigma_{h} .
$$

Moreover, using arguments similar to those of Section 4 we have the following existence and uniqueness results:

THEOREM 5.1. Let (2.7)-(2.9) hold. Then there exists a unique solution $u_{h}$ of problem $P_{1}^{h}$ having the regularity $u_{h} \in V$.

THEOREM 5.2. Let (2.7)-(2.9) hold. Then there exists a unique solution $\sigma_{h}$ of problem $P_{2}^{h}$ having the regularity $\sigma_{h} \in \mathcal{H}_{1}$.

REMARK 5.3. Using the subdifferentiability of the functional $j$ on $V$, an equivalence result similar to Theorem 4.3 may be proved in order to point out the link between the solutions $u_{h}$ and $\sigma_{h}$ obtained in Theorems 5.1 and 5.2. For this reason we shall consider in the sequel the couple $\left\{u_{h}, \sigma_{h}\right\}$ as a weak solution for the mechanical problem (5.1)-(5.5).

The weak solution of (5.1)-(5.5) depends on the parameter $h>0$. The behaviour of this solution as $h \rightarrow 0$ is given by the following theorem.

Theorem 5.4. Let (2.7)-(2.9) hold. For all $h>0$ let $u_{h}$ denote the solution of the variational problem $P_{1}^{h}$ and let $\sigma_{h}$ be the solution of the 
variational problem $P_{2}^{h}$. Then

$$
\begin{array}{ll}
u_{h} \rightarrow u & \text { in } V, \\
\sigma_{h} \rightarrow \sigma & \text { in } \mathcal{H}_{1},
\end{array}
$$

as $h \rightarrow 0$, where $u$ is the solution of problem $P_{1}$ and $\sigma$ is the solution of problem $P_{2}$.

Pr o of. Taking $v=0$ in (5.9) we obtain

$$
\left\langle F\left(\varepsilon\left(u_{h}\right)\right), \varepsilon\left(u_{h}\right)\right\rangle_{\mathcal{H}}+\frac{1}{h} j\left(u_{h}\right) \leq\left\langle f, u_{h}\right\rangle_{V} \quad \forall h>0
$$

and using (2.7) and (2.3), after some algebra we deduce that

$$
\left(u_{h}\right) \text { is a bounded sequence in } V \text {. }
$$

Therefore, there exists $\widetilde{u} \in V$ such that, passing to a subsequence again denoted by $\left(u_{h}\right)$, we have

$$
u_{h} \rightarrow \widetilde{u} \text { weakly in } V \text {. }
$$

Using now (5.13), (5.14) and (2.7) we obtain

$$
j\left(u_{h}\right) \leq C h \quad \forall h>0
$$

where $C>0$ does not depend on $h$. So, by (5.15) and (5.16) it follows that

$$
j(\widetilde{u}) \leq \liminf _{h} j\left(u_{h}\right)=0 .
$$

Having in mind (5.7), (5.6) we obtain $\widetilde{u}_{\nu} \leq 0$ a.e. on $\Gamma_{3}$ and by (2.11) it follows that

$$
\widetilde{u} \in U
$$

Moreover, taking $v=\widetilde{u}$ in (5.9) and using (5.17) we obtain

$$
\left\langle F\left(\varepsilon\left(u_{h}\right)\right), \varepsilon\left(u_{h}\right)-\varepsilon(\widetilde{u})\right\rangle_{\mathcal{H}} \leq\left\langle f, u_{h}-\widetilde{u}\right\rangle_{V} \quad \forall h>0
$$

and (5.15) gives

$$
\limsup _{h}\left\langle F\left(\varepsilon\left(u_{h}\right)\right), \varepsilon\left(u_{h}\right)-\varepsilon(\widetilde{u})\right\rangle_{\mathcal{H}} \leq 0 .
$$

Using now $(2.7)(\mathrm{b})$ we deduce

$$
\left\langle F\left(\varepsilon\left(u_{h}\right)\right), \varepsilon\left(u_{h}\right)-\varepsilon(\widetilde{u})\right\rangle_{\mathcal{H}} \geq\left\langle F(\varepsilon(\widetilde{u})), \varepsilon\left(u_{h}\right)-\varepsilon(\widetilde{u})\right\rangle_{\mathcal{H}} \quad \forall h>0
$$

and by (5.15),

$$
\liminf _{h}\left\langle F\left(\varepsilon\left(u_{h}\right)\right), \varepsilon\left(u_{h}\right)-\varepsilon(\widetilde{u})\right\rangle_{\mathcal{H}} \geq 0 .
$$

Therefore, from (5.18) and (5.19) we obtain

$$
\lim _{h}\left\langle F\left(\varepsilon\left(u_{h}\right)\right), \varepsilon\left(u_{h}\right)-\varepsilon(\widetilde{u})\right\rangle_{\mathcal{H}}=0 .
$$



by

Let now $v \in V$ and let $\theta \in(0,1)$. We denote by $w$ the element of $V$ given

$$
w=(1-\theta) \widetilde{u}+\theta v .
$$

Using (2.7.)(b) and (5.21) shows that

$$
\left\langle F\left(\varepsilon\left(u_{h}\right)\right)-F(\varepsilon(w)), \varepsilon\left(u_{h}\right)-\varepsilon(\widetilde{u})+\theta(\varepsilon(\widetilde{u})-\varepsilon(v))\right\rangle_{\mathcal{H}} \geq 0,
$$

which implies

$$
\begin{aligned}
\left\langle F\left(\varepsilon\left(u_{h}\right)\right), \varepsilon\left(u_{h}\right)-\varepsilon(\widetilde{u})\right\rangle_{\mathcal{H}}+\theta\left\langle F\left(\varepsilon\left(u_{h}\right)\right), \varepsilon(\widetilde{u})-\varepsilon(v)\right\rangle_{\mathcal{H}} \\
\geq\left\langle F(\varepsilon(w)), \varepsilon\left(u_{h}\right)-\varepsilon(\widetilde{u})\right\rangle_{\mathcal{H}}+\theta\langle F(\varepsilon(w)), \varepsilon(\widetilde{u})-\varepsilon(v)\rangle_{\mathcal{H}} .
\end{aligned}
$$

Using now (5.20), (5.15) and (5.22) we obtain

$$
\liminf _{h}\left\langle F\left(\varepsilon\left(u_{h}\right)\right), \varepsilon(\widetilde{u})-\varepsilon(v)\right\rangle_{\mathcal{H}} \geq\langle F(\varepsilon(w)), \varepsilon(\widetilde{u})-\varepsilon(v)\rangle_{\mathcal{H}} .
$$

Moreover, since

$$
\begin{aligned}
\left\langle F\left(\varepsilon\left(u_{h}\right)\right), \varepsilon\left(u_{h}\right)-\varepsilon(v)\right\rangle_{\mathcal{H}}= & \left\langle F\left(\varepsilon\left(u_{h}\right)\right), \varepsilon\left(u_{h}\right)-\varepsilon(\widetilde{u})\right\rangle_{\mathcal{H}} \\
& +\left\langle F\left(\varepsilon\left(u_{h}\right)\right), \varepsilon(\widetilde{u})-\varepsilon(v)\right\rangle_{\mathcal{H}},
\end{aligned}
$$

from (5.20) and (5.23) we deduce

$$
\liminf _{h}\left\langle F\left(\varepsilon\left(u_{h}\right)\right), \varepsilon\left(u_{h}\right)-\varepsilon(v)\right\rangle_{\mathcal{H}} \geq\langle F(\varepsilon(w)), \varepsilon(\widetilde{u})-\varepsilon(v)\rangle_{\mathcal{H}} .
$$

Using now (5.21), (2.7) and taking the limit in (5.24) as $\theta \rightarrow 0$ leads to

$$
\begin{aligned}
\liminf _{h}\left\langle F\left(\varepsilon\left(u_{h}\right)\right), \varepsilon\left(u_{h}\right)-\varepsilon(v)\right\rangle_{\mathcal{H}} & \\
& \geq\langle F(\varepsilon(\widetilde{u})), \varepsilon(\widetilde{u})-\varepsilon(v)\rangle_{\mathcal{H}} \quad \forall v \in V .
\end{aligned}
$$

Again by (5.9),

$\frac{1}{h} j(v)-\frac{1}{h} j\left(u_{h}\right) \geq\left\langle f, v-u_{h}\right\rangle_{V}+\left\langle F\left(\varepsilon\left(u_{h}\right)\right), \varepsilon\left(u_{h}\right)-\varepsilon(v)\right\rangle_{\mathcal{H}} \quad \forall h>0, v \in V$ and having in mind (2.11) and (5.7) we obtain

$$
\left\langle F\left(\varepsilon\left(u_{h}\right)\right), \varepsilon\left(u_{h}\right)-\varepsilon(v)\right\rangle_{\mathcal{H}}+\left\langle f, v-u_{h}\right\rangle_{V} \leq 0 \quad \forall v \in U, h>0 .
$$

Therefore, from (5.25), (5.26) and (5.15) it results that

$$
\langle F(\varepsilon(\widetilde{u})), \varepsilon(v)-\varepsilon(\widetilde{u})\rangle_{\mathcal{H}} \geq\langle f, v-\widetilde{u}\rangle_{V} \quad \forall v \in U .
$$

From (5.17) and (5.27) we find that $\widetilde{u}$ is a solution of the variational inequality (3.7) and from the uniqueness part of Theorem 4.1 we obtain $\widetilde{u}=u$. Since $u$ is the unique weak limit of any subsequence of $\left(u_{h}\right)$, we deduce that the whole sequence $\left(u_{h}\right)$ is weakly convergent in $V$ to $u$ :

$$
u_{h} \rightarrow u \quad \text { weakly in } V \text { as } h \rightarrow 0 .
$$


In order to obtain the strong convergence, note that from (2.7) and (2.3) it follows that

$$
\begin{aligned}
C\left|u_{h}-u\right|_{V}^{2} \leq & \left\langle F\left(\varepsilon\left(u_{h}\right)\right), \varepsilon\left(u_{h}\right)-\varepsilon(u)\right\rangle_{\mathcal{H}} \\
& -\left\langle F(\varepsilon(u)), \varepsilon\left(u_{h}\right)-\varepsilon(u)\right\rangle_{\mathcal{H}} \quad \forall h>0
\end{aligned}
$$

where $C>0$ does not depend on $h$. Putting now $v=u$ in (5.9) we obtain

$$
\left\langle F\left(\varepsilon\left(u_{h}\right)\right), \varepsilon\left(u_{h}\right)-\varepsilon(u)\right\rangle_{\mathcal{H}} \leq\left\langle f, u_{h}-u\right\rangle_{V} \quad \forall h>0 .
$$

The strong convergence result (5.11) follows now from (5.28)-(5.30).

Let us now prove (5.12). For this we remark that from Theorem 4.3 we obtain

$$
\sigma=F(\varepsilon(u)) \quad \text { a.e. in } \Omega
$$

and from (3.7) it follows that

$$
\operatorname{Div} \sigma+\varphi_{1}=0 \quad \text { a.e. in } \Omega \text {. }
$$

In a similar way (see Remark 5.3) we have

$$
\sigma_{h}=F\left(\varepsilon\left(u_{h}\right)\right) \quad \text { a.e. in } \Omega
$$

and from (5.9) it results that

$$
\text { Div } \sigma_{h}+\varphi_{1}=0 \quad \text { a.e. in } \Omega \text {. }
$$

Therefore, by (5.31)-(5.34) we deduce

$$
\left|\sigma_{h}-\sigma\right|_{\mathcal{H}_{1}}=\left|\sigma_{h}-\sigma\right|_{\mathcal{H}}=\left|F\left(\varepsilon\left(u_{h}\right)\right)-F(\varepsilon(u))\right|_{\mathcal{H}} .
$$

The strong convergence result (5.12) follows now from (5.35), (2.7), (5.11).

REMARK 5.5. The mechanical interpretation of the above strong convergence result is the following: the weak solution $\{u, \sigma\}$ of the problem (1.1)-(1.5) modelling the frictionless contact between the elastic body and a rigid foundation may be obtained as the strong limit of the weak solutions $\left\{u_{h}, \sigma_{h}\right\}$ of the problem (5.1)-(5.5) which model the contact between the elastic body and a deformable foundation, when the deformability coefficient of the foundation tends to zero.

\section{References}

[1] H. Brezis, Equations et inéquations non linéaires dans les espaces vectoriels en dualité, Ann. Inst. Fourier (Grenoble) 18 (1968), 115-175.

[2] M. Burguera and J. M. Viaño, Numerical solving of frictionless contact problems in perfectly plastic bodies, Comput. Methods Appl. Mech. Engrg. 121 (1995), 303-322.

[3] S. Drabla, M. Rochdi and M. Sofonea, On a frictionless contact problem for elastic-viscoplastic materials with internal state variables, Math. Comput. Modelling 26 (1997), no. 12, 31-47. 
[4] G. Duvaut et J. L. Lions, Les Inéquations en Mécanique et en Physique, Dunod, Paris, 1972.

[5] G. Fichera, Boundary value problem of elasticity with unilateral constraints, Encyclopedia of Physics, S. Flugge (ed.), Vol. VI a/2, Springer, Berlin, 1972.

[6] J. Haslinger and I. Hlaváček, Contact between elastic bodies. I. Continuous problem, Appl. Math. 25 (1980), 324-347.

[7] -, - Contact between elastic perfectly plastic bodies, ibid. 27 (1982), 27-45.

[8] I. Hlaváček and J. Nečas, Mathematical Theory of Elastic and Elastoplastic Bodies: an Introduction, Elsevier, Amsterdam, 1981.

[9] - - - Solution of Signorini's contact problem in the deformation theory of plasticity by secant modules method, Appl. Math. 28 (1983), 199-214.

[10] I. R. Ionescu and M. Sofonea, Functional and Numerical Methods in Viscoplasticity, Oxford Univ. Press, Oxford, 1993.

[11] N. Kikuchi and J. T. Oden, Theory of variational inequalities with application to problems of flow through porous media, Internat. J. Engrg. Sci. 18 (1980), 1173-1284.

[12] -, - Contact Problems in Elasticity, SIAM, Philadelphia, 1988.

[13] P. D. Panagiotopoulos, Inequality Problems in Mechanics and Applications, Birkhäuser, Basel, 1985.

[14] M. Rochdi and M. Sofonea, On frictionless contact between two elastic-viscoplastic bodies, Quart. J. Mech. Appl. Math. 50 (1997), 481-496.

[15] M. Sofonea, On a contact problem for elastic-viscoplastic bodies, Nonlinear Anal. 29 (1997), 1037-1050.

Institute of Mathematics

University of Setif

19000 Setif, Algeria

Department of Mathematics

University of Perpignan

52 Avenue de Villeneuve

66860 Perpignan, France

E-mail: sofonea@univ-perp.fr
Institute of Mathematics

University of Constantine 25000 Constantine, Algeria

Reçu par la Rédaction le 3.12 